\title{
SYSTÈMES ÉCRIVANTS ET RÉPARTITION DES RÔLES INTERACTIONNELS
}

\begin{abstract}
A bstract. Krafft Ulrich, Dausendschön-Gay Ulrich, Systèmes écrivants et répartition des rôles interactionnels [Writing systems and repartition of interactional roles]. Studia Romanica Posnaniensia, Adam Mickiewicz University Press, Poznań, vol. XXV/XXVI: 2000, pp. 199-212, ISBN 83-232-0965-0, ISSN 0137-2475.
\end{abstract}

In cooperative writing two or more interacting persons work together to write a common text. After several case studies with unplanned writing interactions, in an experiment we asked 17 dyads to compose a recipe for mousse au chocolat. In the first part of the essay we show that one can describe the cooperating dyads as writing systems with specific properties, the interacting persons as agents of the system. After that we ask how far the writing system determines the activities of its agents and to what extent the distribution of activities, conversely, follows the wishes and preferences of those interacting. We also examine whether one can speak of a "writing style" of the writers working together in the writing system.

Dans le vaste domaine de l'analyse de l'interaction sociale par son côté verbal', nous avons choisi de travailler sur des communications qui ont un but précis et fixé au préalable et qui, par là, sont différentes des situations de small talk. Dans le cadre de ces recherches, nous avons commencé à étudier des corpus de rédactions conversationelles ${ }^{2}$ pour mieux cerner le rapport entre les procédés (observables dans le discours) de la production verbale et les paramètres situationnels (tels les individus, les

${ }^{1}$ Nos recherches relèvent de ce qu'on appelle l'analyse conversationnelle linguistique. Cf. Gülich 1991 et Heritage 1995 pour un résumé des principales notions et conceptions. Cf. Gülich, Kotschi 1995 pour l'analyse de la production verbale à partir de données discursives.

${ }^{2}$ Nous avons appelé rédactions conversationnelles des situations où deux ou plusieurs personnes se mettent autour d'une table pour concevoir et rédiger un texte commun. La spécificité de ces interactions est multiple: Les interactants se soumettent aux contraintes d'un travail collectif; leur travail vise un résultat précis et matériel (un texte inscrit sur du papier ou dans un fichier électronique); le travail est limité temporellement, par l'achèvement du texte; chaque interactant assume l'entière responsabilité pour le produit commun. Néanmoins, cette situation rédactionnelle est conversationnelle dans ce sens que les échanges verbaux ne sont pas préconçus (et imprévisibles) et que l'organisation de l'interaction est négociée librement parmi les participants. Cf. Dausendschön-Gay, Gülich, Krafft 1992. 
objectifs et motifs, l'espace et le temps). Nos premières observations et hypothèses se sont développées à partir de corpus de rédactions de lettres officielles, de mémoires linguistiques ou de rapports officiels, qui avaient tous l'avantage d'être authentiques et le désavantage de ne pas être comparables de par la spécificité des tâches, situations et personnes. Méthodologiquement, il s'agissait d' études de cas.

L'expérimentation de la «mousse au chocolat» ${ }^{3}$ offre une alternative méthodologique. Comme les 17 dyades qui ont participé à l'expérience ont travaillé dans les mêmes conditions, avec la même instruction et en envisageant le même résultat, nous pouvons comparer les procédés qu'ils choisissent pour résoudre les problèmes spécifiques de la tâche; comme chacun des trois sous-groupes de l'expérience a le statut de groupe-contrôle par rapport aux deux autres, nous pouvons étudier l'influence du paramètre linguistique (par exemple l'influence de la L1 sur la production en L2) sur les procédés employés par les dyades.

La comparaison des corpus a fait apparaître en particulier que non seulement les paramètres situationnels sont relativement stables dans l'expérimentation, mais que les dyades agissent plus ou moins selon les mêmes principes puisqu'on observe des «comportements de dyade» assez comparables. Pour mieux cerner les phénomènes récurrents dans le travail de toutes les dyades, nous avons trouvé important d'adopter une perspective analytique supplémentaire qui n'est pas inhérente à l'analyse conversationnelle: nous parlerons moins de personnes qui accomplissent des activités, mais plutôt de «tâches à accomplir dans le système à rédiger un texte» que nous appelerons le système écrivant. Nous empruntons cette façon de parler et de voir à la théorie des systèmes qui essaie, pour certains phénomènes de l'interaction sociale, de décrire la fonctionalité d'une action dans le cadre d'un système destiné à lever un problème;

\footnotetext{
${ }^{3}$ Nous avons demandé à 17 dyades de rédiger, sous l'oeil d'une caméra vidéo, la recette de la mousse au chocolat. L'idée était de construire une expérimentation qui permette aux participants de concentrer toute leur attention sur le processus de la mise en mots de contenus donnés; il ne s'agissait pas pour eux de discuter le mode de confection du plat, mais de rédiger la recette d'après la présentation vidéo. La tâche, c'était de produire une «vraie» recette, qui fonctionnerait donc sans la vidéo qui montre les étapes successives de la confection d'une mousse au chocolat. Les participants (des dyades de personnes qui se connaissent bien avant l'expérimentation et qui sont venues ensemble) peuvent regarder la vidéo autant de fois qu'ils le veulent, après l'instruction orale que nous leur avons donnée au début. Les dyades disposent en plus d'une feuille de papier contenant la version écrite de l'instruction, la liste des ingrédients et un espace vide pour l'inscription du texte. Les dyades se composent de trois groupes différents: 1) participants de langue première ( $\mathrm{Ll}$ ) différente (par exemple une coréenne et un chinois) qui communiquent et rédigent en allemand (leur $\mathrm{L} 2$ ); 2) participants de $\mathrm{L} 1$ identique (par exemple deux françaises ou deux allemands) qui communiquent dans leur langue commune mais rédigent en L2 (en l'occurrence en allemand ou en français); 3) participants de Ll identique qui communiquent et rédigent en L1. Un enregistrement vidéo assure la documentation de toutes les interactions, de l'entrée dans la salle jusqu'à la fin de l'expérimentation. Chacun participe à une courte séance de rétrospection individuelle immédiatement après la séance de rédaction. Nous disposons en outre de questionnaires que chaque participant a remplis avant la séance et qui leur demandent, outre quelques données personnelles, l'auto-évaluation de leurs compétences culinaires, le cas échéant des compétences linguistiques en L2 ainsi que des renseignements sur leurs expériences rédactionnelles préalables.
} 
cette théorie s'intéresse surtout à la coordination des activités à l'intérieur de ce système en vue de leur apport à la solution du problème ${ }^{4}$.

Dans ce qui suit, nous discuterons le problème du rapport entre l'individu et le système dont il est un «agent». Pour ce faire, nous décrirons d'abord brièvement les constituant du système écrivant ${ }^{5}$. Puis nous essaierons de réintroduire l'individu dans l'analyse en demandant dans quelle mesure le système détermine les activités de ses agents, et dans quelle mesure ceux-ci sont libres de choisir les activités qu'ils préfèrent.

\section{LA RÉDACTION CONVERSATIONNELLE EST L'ACTIVITÉ D'UN SYSTÈME ÉCRIVANT}

\section{1. ÉPISODE SOCIAL}

Toutes les dyades entreprennent au début de l'expérimentation plusieurs activités qui nous rapellent la notion de cadrage d'Erving Goffman, et dont la fonction est de réaliser les conditions qui constituent l'interaction rédactionnelle en tant qu'épisode social. Nous comptons parmi ces activités en particulier celles qui définissent l'espace interactionnel du travail collectif dans ses dimensions spatiale et temporelle, ainsi que les activités de figuration.

Au moment d'entrer dans la salle, les interactants des différentes expérimentations regardent autour d'eux pour s'orienter dans cet espace qui leur est inconnu. Mais dès l'instruction pour l'expérience, au plus tard avec le début du travail collectif (première vision de l'instruction vidéo), toutes les dyades définissent par l'orientation de leurs regards et la position de leurs corps l'espace et les objets pertinents pour leur collaboration: l'écran de l'installation vidéo (qu'ils regardent); le lecteur vidéo (qu'ils manipulent); les objets sur la table (qu'ils emploient), en particulier la feuille qui porte la liste des ingrédients et où il faudra inscrire le texte de la recette; les interactants eux-mêmes qui se placent de façon à former une sorte de demi-cercle, esquissant ainsi le territoire de la dyade. Tous les regards et les gestes sont orientés à l'intérieur de cet espace; même la "position zéro» du regard pendant les périodes de réflexion ne dépasse pas ses limites imaginaires. Ce n'est qu'au moment d'interruptions (questions des chercheurs, bruits de personnes qui passent devant la porte et qui suspendent le travail en cours) que les interactants prennent en compte le monde extérieur à l'es-

${ }^{4}$ Cette notion de système est indépendante du nombre d'agents qui participent au travail collectif, et en principe elle est aussi indépendante de la qualité des agents (être humain ou machine programmée). Il faut cependant souligner, pour éviter des malentendus, que le système «n'existe pas» en dehors de la situation et des personnes qui la créent. Le système est une construction permanente de ses acteurs qui les lie tant qu'ils sont en train d'achever un travail précis qui est organisế à l'intérieur de ce système flexible.

${ }^{5}$ Cf. pour une analyse plus détaillée Krafft, Dausendschön-Gay (sous presse). 
pace interactionnel. Après la fin du travail collectif, tout change de nouveau: les regards s'orientent au-delà de l'espace interactionnel, les interactants appellent les chercheurs ("nous avons fini») ou commencent à fouiller dans leurs sacs, à bavarder, à se montrer des livres ou dossiers qu'ils ont apportés pour la journée, etc.

La dimension temporelle est définie par les interactants pour les besoins de leur collaboration. Il y a d'abord la consigne de l'expérience qui propose 15 minutes pour terminer le travail et qui influe par moments sur les actions (regards vers la montre, rappels du temps déjà écoulé, refus de séquences latérales hors propos). Le travail prend un certain rythme - du moins pour les dyades qui arrivent à bien coordonner leurs activités - ce qui comporte un consensus implicite sur la longueur des silences que les dyades sont prêtes à accepter tant que leur apport au travail semble assuré (phases de réflexion, consultation du dictionnaire, relecture du texte déjà inscrit, etc.).

Il est d'autre part indispensable à la situation sociale du travail que les interactants se manifestent mutuellement le respect de leurs faces positives et négatives; avec Goffman, on dirait qu'on ne peut pas ne pas le faire. Les petites séquences de figuration ne sont pas rares, comme celle de la dyade 15 quand les deux interactantes disent en même temps «stop» pour arrêter la vidéo: un petit rire commun, un sourire et un regard mutuel de deux secondes suffisent pour confirmer l'ambiance amicale de l'épisode. Quand la situation s'y prête ou le demande (petits conflits, désaccords), le travail de figuration se fait pour permettre aux participants de se séparer à la fin sur un mode de rapport social qui corresponde à celui avec lequel ils sont entrés dans l'interaction. Il est d'ailleurs important de remarquer que la qualité du rapport social entre les agents du travail rédactionnel influe directement sur la qualité du travail.

\subsection{OBJECTIF}

Avant que le système puisse déterminer les types d'activités et leur ordre, il faut qu'il définisse l'objectif et le résultat de l'interaction ainsi que les modalités de collaboration. Nous avons développé les notions de tâche conversationnelle et de contrat pour décrire ces activités de cadrage au début d'un épisode ${ }^{6}$. À peu d'exceptions près, les dyades de l'expérimentation verbalisent à voix haute ce qu'elles voient pendant la première vision de la bande vidéo, une activité qui est absente des pré-tests avec des rédacteurs solitaires qui travaillaient sous les mêmes consignes que les dyades. À côté d'autres explications de ce phénomène (recherche lexicale chez les dyades exolingues par exemple), il est évident que ces verbalisations proposent une interprétation que les interactants font de la vidéo et demandent une ratification réciproque; c'est ainsi que l'objet verbalisé (qui est différent de l'objet perçu!) est construit dans l'interaction. Le travail collectif ne peut pas faire l'économie de la constitution de ce que Clark 1996 appelle le «common ground», quel que soit le degré d'univocité de la consigne. Nous observons en plus que le même procédé se répète au début de

${ }^{6}$ Cf. Dausendschön-Gay, Krafft 1991 et Krafft, Dausendschön-Gay 1993. 
chaque nouvelle séquence rédactionnelle: vision de la vidéo (et/ou lecture des notes et/ou récupération mémorielle) avec une première verbalisation, puis début du travail rédactionnel.

Le contrat de rédaction une fois conclu, il faut prendre d'autres décisions de portée générale sans lesquelles le travail ne peut commencer: l'encadrement du texte (avec ou sans titre, avec ou sans formule de clôture) et sa structure globale (énumération des étapes de la confection avec des tirets ou plutôt texte cohérent à phrases complètes et complexes; format syntaxique des phrases: infinitifs, impératifs, etc.). Tout ceci doit se régler avant ou au moment de la production de la première phrase. Les choix se font souvent de façon implicite et sans négociation entre les interactants, et il est rare que ceux-ci reviennent sur leurs décisions. Toutes ces activités sont destinées à définir les qualités formelles du texte qui sera le résultat du travail? ${ }^{7}$.

\subsection{RESSOURCES MATÉRIELLES}

Les ressources matérielles (l'instruction vidéo, le papier, les stylos, le dictionnaire) ne sont pas simplement présentes sur la scène en tant que telles, mais les interactants, en les employant, leur donnent un statut spécifique à l'intérieur du système écrivant.

Prenons l'exemple de l'instruction vidéo: Les dyades 11,13 et 15 par exemple choisissent pour leur travail rédactionnel un modèle strictement séquentiel qui fait que la rédaction est organisée selon les conditions introduites par l'instruction vidéo: elles regardent l'une après l'autre les séquences de la confection telles que la vidéo les présente, arrêtant la bande à chaque fin de séquence pour concevoir la phrase correspondante; ainsi, les 19 séquences de la vidéo sont-elles rendues en 16 phrases (ou plutôt constructions verbales) par la dyade $11^{8}$.

La dyade 4 procède différemment. Chacune des interactantes prend des notes pendant le premier passage de la bande vidéo. Pendant la première partie de la rédaction" elles ne regardent plus la vidéo, mais conçoivent leur texte à partir de ce qu'elles ont mémorisé et avec l'aide des notes qui se retrouvent parfois mot à mot dans le texte inscrit qui contient moins de constructions verbales que celui de la dyade 11 et dont les phrases sont plus complexes.

${ }^{7}$ Nous avons développé l'idée du «modèle de texte» dans Krafft, Dausendschön-Gay 1996. Les décisions prises au départ acquièrent une force normative, même si le degré d'obligation qu'elles établissent n'est pas absolu. Les choix peuvent être révoqués si les conséquences qui en résultent sont contra-productrices ou trop difficiles à réaliser.

${ }^{8}$ On peut faire le même type d'observations pour l'emploi que les dyades font du dictionnaire: les dyades 13 et 15 ne le prennent jamais en considération (il ne fait pas partie de leur espace interactionnel, bien que «visiblement» sur la table), les interactantes de la dyade 10 en font un objet primordial de consultation et d'organisation du travail, le partenaire $B$ de la dyade 11 se perd totalement dans des consultations qui n'ont plus rien à voir avec le travail en cours.

${ }^{9}$ La dyade 4 change son procédé après la moitié du travail, parce que les notes ne suffisent plus à reconstruire l'ordre des activités de la confection. 


\subsection{NORMES ET CONVENTIONS}

Métaphoriquement parlant on pourrait dire d'un côté que les agents du système écrivant s'approprient les ressources matérielles pour les exploiter dans des processus cognitifs, mais que d'autre part les interactants arrivent avec un bagage de savoirs qu'ils mettent à la disposition du système pendant le travail. Nous trouvons des manifestations de compétences linguistiques générales dans les évaluations sur la correction d'un énoncé ou dans les appréciations stylistiques qui aident à choisir une forme linguistique plutôt qu'une autre; nous relevons aussi les traces d'une compétence rédactionnelle générale et de l'expertise culinaire des partenaires. Nous remarquons que les solutions de problèmes dépendent très souvent des normes de l'activité rédactionnelle et des conventions sociales sur le type de texte à écrire. C'est à partir de ces savoirs que les interactants vont décider par exemple de mettre un titre entre la liste des ingrédients et le texte de la recette. Le même type de savoirs se manifeste dans le choix du format syntaxique des phrases: les alternatives n'étant pas légion, il n'y a que les versions infinitif, impératif ou phrases assertives et ceci dans des textes énumératifs (tirets ou numérotage des étapes). De la même façon, l'instruction de l'expérimentation développe, elle aussi, une force normative pour le travail; ceci en particulier en ce qui concerne l'orientation vers les destinataires de la recette qui n'ont pas été définis de façon très précise dans l'instruction. Nous participons assez souvent à des négociations qui tournent autour de la question de savoir si les lecteurs vont comprendre telle expression et s'ils seront capables d'inférer telle étappe de la confection.

\subsection{TÂCHES}

Les tâches appartiennent à différents domaines et appellent différents types d'activités:

- La tâche sociale de la constitution et du maintien du système écrivant se manifeste dans la gestion spatiale et temporelle de l'espace interactionnel et dans les activités de figuration;

- De l'objectif de l'interaction, la rédaction d'une recette de cuisine, découlent différentes tâches de production: définition de l'objet à décrire, conception des qualités du texte, verbalisation, recherche lexicale, mise en mots, inscription, contrôle de la correction du texte inscrit ${ }^{10}$.

- Le bon fonctionnement du système demande un travail d'organisation qui coordonne les activités des différents domaines (Dausendschön-Gay et al. 1992).

${ }^{10} \mathrm{Cf}$. au sujet des activités de production et de leur organisation en phases Dausendschön-Gay, Gülich, Krafft 1992 et Bouchard, de Gaulmyn 1997. 
Les activités correspondant à ces tâches sont des composantes du système écrivant; elles ne sont liées ni à une personne ni à un accord préalable entre les interactants ni aux consignes particulières de l'expérimentation. Celui qui assume - momentanément ou à long terme - la responsabilité d'une de ces activités le fait en sa qualité d'agent du système. En effet, la prise en charge d'une activité dépend souvent de la situation locale, actuelle de la dyade, les décisions étant prises ad hoc et indépendemment des compétences que les interactants peuvent apporter pour une activité spécifique. De mềme que rien ne permet de prévoir la personne qui va accomplir l'activité nécessaire, il est impossible de distinguer l'apport des interactants au résultat final. L'absence de rôles fixés une fois pour toutes et la circulation des responsabilités sont des arguments importants à l'appui de la thèse qui place le système écrivant au centre de l'analyse et considère les interactants d'abord comme agents du système.

\section{LA RÉPARTITION DES ACTIVITÉS DANS LES SYSTÈMES ÉCRIVANTS}

Si les interactants sont d'abord des agents d'un système qui demande d'accomplir des tâches et de résoudre des problèmes, il n'en reste pas moins à résoudre le problème de la répartition des activités. Nous venons de constater que les systèmes écrivants de la mousse ne fixent pas de rôles. Ceci n'est pas vrai pour tous les systèmes: il existe des systèmes qui prévoient une répartition des activités, c'est-à-dire des rôles ${ }^{11}$.

\subsection{SYSTĖMES ÉCRIVANTS À RÔLES PRÉDÉFINIS}

Les systèmes écrivants à répartition d'activités définie sont des systèmes qui se constituent autour de la mise en contact de différentes expertises. Un cas fréquent et que nous retrouvons plusieurs fois dans nos corpus: une allemande a besoin d'écrire une lettre officielle en français (pour poser une candidature, demander un service etc.); elle demande à une personne de langue maternelle française de l'aider, lui soumettra peut-être un brouillon ou une première version de la lettre. Le système écrivant va fonctionner selon une règle de répartition des activités qui distinguera deux rôles: la personne experte en langue sera responsable de tout ce qui concerne la forme linguistique du texte à produire: correction morphologique, style épistolaire, mise en page etc., et elle aura le droit et l'obligation d'initier dans ce domaine toutes les activités qu'elle jugera nécessaires. L'auteur officiel de la lettre sera responsable du contenu; son rôle sera d'informer le système et de contrôler, du point de vue du contenu, le résultat des activités linguistiques de son partenaire ${ }^{12}$.

"Nous définissons les rôles dans les interactions comme des règles de répartition des activités. Cf. Dausendschön-Gay, Krafft 1991 .

12 Cf. pour l'analyse de ce type de rédactions conversationnelles Dausendschön-Gay, Gülich, Krafft 1992 et Bouchard, de Gaulmyn 1997. 


\section{2. «STYLES RÉDACTIONNELS» DANS LES SYSTĖMES ÉCRIVANTS SANS RÔLES PRÉDÉTERMINÉS}

Les systèmes écrivants qui produisent la recette de la mousse au chocolat ne prévoient aucune définition de rôles a priori. Le système donne des tâches à accomplir, il demande aux interactants d'agir, mais il ne dit rien sur la répartition des activités. On pourrait donc penser que cette répartition se fait essentiellement selon les préférences individuelles des partenaires, et lorsqu'en analysant les interactions, on constate par exemple que certaines personnes s'occupent plus particulièrement de la correction morphologique ou veulent expliciter tous les détails de la description des opérations culinaires, nous sommes tentés de voir dans ces comportements des choix ou des styles de production. Cette interprétation psychologisante sera même la seule plausible tant que nous travaillerons, dans des études de cas, sur des interactions isolées.

Voyons par exemple la dyade 12: une Hongroise $(\mathrm{H})$ et une Malgache $(\mathrm{M})$ rédigent en allemand. L'analyse permet de dégager pour chacune des partenaires par rapport à différentes tâches rédactionnelles des comportements spécifiques qui semblent obéir à ce qu'on pourrait appeler, en exploitant la terminologie de Goffman, une «ligne de conduite rédactionnelle». Voici des éléments de cette ligne de conduite dans quatre tâches rédactionnelles ${ }^{13}$ :

(i) Il faut - c'est une tâche rédactionnelle - rédiger un texte linguistiquement correct. Dès le début, $\mathrm{H}$ fait savoir qu'elle considère $\mathrm{M}$ comme plus compétente en allemand; au cours de l'interaction, elle répète plusieurs fois cette appréciation, sans que $\mathrm{M}$ ne la contredise jamais. A cette évaluation des compétences correspond le fait que $\mathrm{M}$ prenne la responsabilité de la correction (surtout morphologique) du texte. Elle contrôle et, le cas échéant, décide.

(ii) Les instructions précisent que la recette doit permettre de confectionner la mousse sans l'aide de la vidéo. La dyade va donc devoir choisir les détails nécessaires à la bonne compréhension des opérations culinaires. Ce choix se fait en général par un procédé d'amplification progressive: la dyade produit d'abord une version rudimentaire qu'elle enrichit peu à peu jusqu'à ce qu'elle tombe d'accord sur une version suffisamment explicite ${ }^{14}$. Dans l'exemple suivant, on voit comment $\mathrm{H}$ et $\mathrm{M}$ collaborent, chacun apportant des éléments à la phrase inscrite:

Exemple 1: Explicitation par enrichissement progressif (dyade 12; transcription très simplifiée) ${ }^{15}$

\section{M: NOMME NOUVEAU SUJET butter du / le beurre \\ H: PROPOSE IERE VERSION dann butter vorsichtig zugeben puis ajouter du beurre avec précaution}

${ }^{13}$ Nous résumons dans ce qui suit des analyses plus détaillées dans Da usendschön-Gay, Krafft 1996 et Krafft, Dausendschön-Gay 1997.

${ }^{14}$ Pour les détails de ce procédé cf. Krafft, Dausendschön-Gay (sous presse).

15 Conventions de transcription: DICTÉE = commentaire du transcripteur; \# .... \# = début/fin d'un segment inscrit. 

M: AUTODICTÉE nach ein paar minuten butter après quelques minutes, du beurre
$\mathrm{H}$ : DICTÉE die butter le beurre
M: AUTODICTÉE geschmolzene butter beurre fondu
$\mathrm{H}$ : DICTÉE vorsichtig zugeben ajouter avec précaution

Version inscrite: «\#Nach ein Paar Minuten die geschmolzene Butter vorsichtig zugeben» (= Après quelques minutes, ajouter avec précaution le beurre fondu).

Il arrive cependant que les partenaires ne soient pas d'accord, $M$ voulant noter des détails que $\mathrm{H}$ ne juge pas nécessaires. Ces deux tendances peuvent s'opposer jusqu'à provoquer un conflit ${ }^{16}$. Mais même quand les divergences ne vont pas jusque là, c'est typiquement $\mathrm{M}$ qui donne les détails (dans l'exemple 1, elle remplace puis par après quelques minutes et précise que le beurre est fondu) alors que $\mathrm{H}$ se contente d'énoncés simples qui fixent l'information essentielle (dans l'exemple 1, c'est elle qui formule l'énoncé de base puis ajouter du beurre avec précaution) et demande plutôt au lecteur d'exercer ses facultés inférentielles (c'est le sens de la correction le beurre qui assure la cohérence du texte). Ces deux comportements se confirmant à travers toute l'interaction, on peut parler de deux styles rédactionnels, le «style explicitant» et le «style contextuel».

(iii) La mise en mots est une tâche complexe que les interactants n'accomplissent souvent pas d'une traite. Dans l'exemple 1, on distingue trois étapes: $M$ donne le sujet à traiter (beurre), $\mathrm{H}$ propose une première version (puis ajouter du beurre avec précaution) que $\mathrm{M}$ élabore pour aboutir à la version inscrite (Après quelques minutes, ajouter avec précaution le beurre fondu). Cette répartition des activités: première version par $\mathrm{H}$, élaboration par $\mathrm{M}$, fréquente chez cette dyade, est favorisée du fait que c'est $\mathrm{M}$ qui inscrit et que $\mathrm{H}$ commence souvent à chercher une phrase suivante pendant que $\mathrm{M}$ est encore occupée à inscrire la phrase qu'on vient de formuler (ce n'est d'ailleurs pas le cas ici).

Nous voyons se dessiner deux lignes de conduite rédactionnelles: $\mathrm{H}$ propose des premières versions qui formulent les informations essentielles et font confiance au contexte et aux facultés inférentielles du lecteur. $\mathrm{M}$ s'occupe des détails: détails de contenu, détails morphologiques, et au lieu de faire confiance au contexte, elle s'applique à expliciter au maximum. Cette distinction peut donner un sens à une dernière observation:

(iv) La première tâche rédactionnelle est une tâche de compréhension. Les instructions demandent aux interactants de regarder en entier la confection de la mousse. Ils pourront ensuite revenir au début et revoir autant de fois qu'ils le veulent

${ }^{16}$ Dans une séquence trop longue pour la donner ici, où il s'agit de dire qu'il faut remuer le chocolat qu'on vient de faire chauffer au bain-marie, $M$ veut noter que ceci se fera en continuant â faire chauffer au bain-marie, alors que $\mathrm{H}$ estime que cela va de soi. Cf. Dausendschön-Gay, Krafft 1996. 
les différentes phases de la démonstration. La dyade 12 regarde donc comme prévu l'enregistrement, mais en revenant en arrière, ne retrouve pas le début et se perd dans la bande vidéo. À ce moment, $\mathrm{H}$ assure qu'elle a parfaitement compris et propose à plusieurs reprises de rédiger la recette de mémoire. $M$ ne répond pas directement à cette proposition, mais elle s'obstine à chercher le début de l'enregistrement et finit par le trouver (après plusieurs minutes). Encore une fois, $\mathrm{H}$ s'appuie sur la compréhension globale, alors que $M$ veut voir le détail.

Nous pouvons résumer ces observations en distinguant deux styles rédactionnels, en opposant un style «explicitant» à un style «contextuel».

\subsection{PRÉFÉRENCES INDIVIDUELLES ET CONTRAINTES SYSTÉMATIQUES}

Si nous quittons maintenant l'étude d'un cas pour comparer plusieurs systèmes écrivants ${ }^{17}$, il faudra nuancer quelque peu notre analyse, et en particulier les observations que nous avons faites au sujet de la préférence pour l'explicitation s'opposant à la préférence pour le renvoi au contexte et celles qui concernent la distribution des responsabilités pour la correction.

\subsubsection{DÉTERMINER LE DEGRÉ D'EXPLICITATION D'UN SEGMENT}

Nous avons vu que les styles rédactionnels de $\mathrm{H}$ et de $\mathrm{M}$ s'opposent en ce que $M$ veut obtenir un texte explicite alors que $\mathrm{H}$ utilise au contraire les déterminations contextuelles et s'en remet aux inférences du lecteur. Or, nous n'avons trouvé aucune dyade où cette opposition n'apparaisse pas au moins dans une situation. Comme il est très improbable que le hasard ait réuni dans toutes les dyades des représentants d'un style explicitant et d'un style contextuel, il faut voir là une opposition non pas personnelle, mais systématique. Déterminer le degré d'explicitation des segments à construire est en effet une des tâches constantes des dyades, et un des procédés que les interactants utilisent en cas de désaccord dans ce domaine est de confronter des versions plus et moins explicites pour trouver une version satisfaisante. Cette méthode (au sens ethnométhodologique du terme) prévoit que l'un des interactants prenne, le temps de la confrontation, le rôle de l'explicitant, l'autre celui du contextuel. S'il est nécessaire pour un autre segment d'employer encore une fois ce procédé, les interactants pourront choisir de reprendre le même rôle ou au contraire le rôle opposé c'est ce que nous observons dans la majorité des cas. La dyade 12 a ceci de particulier que les interactants reviennent toujours aux mềmes rôles, que $\mathrm{M}$ en particulier semble dans tous les cas proposer et, en cas de désaccord, défendre la version explicite, dans

${ }^{17}$ Pour les analyses suivantes, nous utiliserons des observations faites sur les dyades 12 (une hongroise et une malgache rédigeant en allemand), 17 (deux françaises rédigeant en français), 1,4,11 (allemands rédigeant en français), 13, 15 (françaises rédigeant en allemand). 
le but apparemment de produire, en notant autant de détails que possible, un texte «reader-proof». C'est cette constance que nous percevons comme un style personnel. Nous noterons simplement que ce style se manifeste dans le cadre d'une structure systématique.

\subsubsection{LES RÓLES DU SCRIPTEUR}

Un autre élément de la «ligne de conduite rédactionnelle» de $\mathrm{M}$ est qu'elle prend la responsabilité de la correction du texte. Cette observation prend une toute autre valeur quand on compare les différentes interactions, puisque dans la dyade 12 comme dans toutes les autres dyades, sans exception, c'est la personne qui inscrit (que nous appellerons le «scripteur») qui prend ce rôle. Plus généralement, le scripteur est l'instance qui contrôle le texte qu'il inscrit: contrôle de la correction morphologique, de la complétude du texte, de sa cohérence. Souvent, le scripteur contrôle aussi le rythme de production: en inscrivant rapidement de petits segments, il accélère le rythme, il le ralentit au contraire en attendant pour inscrire que des segments longs soient achevés et contrôlés oralement.

La rareté des ratures témoigne de l'efficacité du travail du scripteur. Nous observons d'autre part qu'un segment change radicalement de statut au moment où il est inscrit: avant l'inscription, il reste disponible pour toutes les modifications possibles, et les partenaires n'hésitent pas à changer un morphème ou à proposer carrément une nouvelle formule; mais dès qu'il est inscrit, on n'y touche plus, sauf en cas d'absolue nécessité $^{18}$. L'inscription est un aboutissement (cf. Bouchard, de Gaulmyn 1997: 158), et pour qu'il puisse l'être, il faut que le scripteur fasse consciencieusement son travail de contrôle.

Comment devient-on donc scripteur? Dans notre expérimentation, les interactants entrent dans la salle sans savoir ce qu'on leur demandera de faire. Ils trouvent une table avec deux chaises, une installation vidéo, sur la table du papier et des crayons. Ce n'est qu'une fois qu'ils sont assis qu'on leur donne leurs instructions, et ce n'est qu'au bout d'un certain temps qu'ils comprennent qu'il faudra manipuler la vidéo et écrire à deux un seul texte. À ce moment, la personne qui se trouve assise à gauche va prendre le rôle de «manipulateur du lecteur vidéo» qui se trouve plus près d'elle, et la personne assise à droite va prendre le rôle complémentaire de scripteur. Le scripteur ayant toujours les mains pleines, c'est la personne de gauche qui va, chez les dyades rédigeant en langue étrangère, compulser le dictionnaire. Autrement dit, c'est tout à fait par hasard qu'un interactant se retrouve scripteur et investi des fonctions de contrôle attachées à ce rôle. Les scripteurs n'en font pas moins consciencieusement leur travail.

${ }^{18}$ Une exception: la dyade 17 déclare dès le début qu'elle produit un brouillon qu'il faudra mettre au propre. Cette dyade ne se prive pas de ratures. 


\subsubsection{SCRIPTEUR ET NON-SCRIPTEUR}

Le travail du scripteur est un travail officiel et publié et qui exerce ainsi une certaine influence sur les activités du non-scripteur: les scripteurs se placent de façon à permettre au non-scripteur de suivre visuellement le travail d'inscription, qu'ils accompagnent très souvent d'une autodictée à voix haute. Le non-scripteur peut profiter de cette invitation et contrôler le travail du contrôleur-scripteur. Mais il arrive qu'il la refuse, soit localement, soit pour toute la durée de l'interaction: le rôle de «contrôleur du contrôleur» est un rôle facultatif.

Un autre type d'influences réciproques entre scripteur et non-scripteur est plus subtil. Les fonctions de contrôle attachées au rôle de scripteur semblent parfois orienter de façon plus générale les activités du scripteur vers le contrôle, ce qui favorise chez le non-scripteur les activités initiatives de mise en mots. Voici un exemple pris à la dyade 4 et assez représentatif pour l'interaction de cette dyade. À partir de la collocation faire du café, A et $\mathrm{O}$ élaborent ensemble la phrase \#\#Faire un grand espresso en prenant quatre cuillers de café\#. La répartition des activités est presque caricaturale: O, non-scripteur, propose des formules; avant d'inscrire, A, scripteur, contrôle et demande des corrections.

Exemple 2: Contrôle et initiative de mise en mots (dyade 4)

O c'est faire du café?

A oui

faire une tasse de café

faire un espresso

faire un grand espresso

faire un grand espresso en prenant

quatre cuillers de café

\section{O contrôle la collocation}

O donne une lère version; $\mathrm{A}$ contrôle sur la liste des ingrédients et demande correction

$\mathrm{O}$ donne une $2 \mathrm{e}$ version; A contrôle sur la liste des ingrédients et suggère une expansion

O donne une $3 \mathrm{e}$ version

A formule, tout en inscrivant, une expansion qui précise «grand» espresso

On voit comment la fonction de contrôle chez le scripteur peut favoriser le rôle d'initiateur de mises en mots chez le non-scripteur. Cette répartition des activités est très nette chez les dyades 12 et 4; chez d'autres dyades, la fréquence des initiatives est plus grande chez le non-scripteur (dyades 1,13,15), alors que nous ne voyons jusqu'à présent aucune dyade où le scripteur serait en même temps l'initiateur principal de mises en mots ${ }^{19}$.

\subsection{PRÉFÉRENCES INDIVIDUELLES ET CONTRAINTES DU SYSTÈME}

Nous avions demandé si et comment le système écrivant, conçu comme l'instance productrice dans la rédaction conversationnelle, exerce une influence sur la répartition des activités, et dans quelle mesure cette répartition répond aux préférences des in-

${ }^{19}$ Mises à part certaines dyades comme la dyade 6 (un chinois et une coréenne rédigeant en allemand) où l'un des partenaires est tellement plus compétent en allemand qu'il fait le travail tout seul. 
teractants, des agents du système. Nous pouvons à présent tenter une première réponse.

Nous avons distingué trois types d'influences que le système écrivant peut exercer sur la répartition des activités:

- Dans les systèmes qui se constituent autour d'expertises divergentes, ces expertises déterminent des rôles interactionnels (par exemple expertise linguistique et expertise de contenu).

- Quand le système comprend un rôle de scripteur ${ }^{20}$, ce rôle semble entraîner directement des responsabilités de contrôle qui concernent en particulier la correction et la complétude du texte. Le non-scripteur peut prendre le rôle de «contrôleur du contrôleur», mais qui reste localement et globalement facultatif. Indirectement, la responsabilité de contrôle peut favoriser chez le scripteur une attitude générale de «contrôleur» et les activités correspondantes, chez le non-scripteur les activités initiatives.

- Les systèmes écrivants disposent de «méthodes» pour résoudre certains problèmes, comme par exemple la confrontation de différentes versions pour trouver le degré d'explicitation convenable. Cette méthode demande de prendre les rôles «d'explicitant» et de «contextuel», qu'on quittera dès que le problème sera résolu.

Ces données du système sont des contraintes pour les interactants-agents. Dans la plupart des cas, les interactants s'y soumettent sans enthousiasme ni dégoût. Mais quand les contraintes systématiques rencontrent la distribution des préférences individuelles, on voit émerger et s'établir des styles interactionnels comme celui de la «contextuelle» $\mathrm{H}$ ou de «l'explicitante» $\mathrm{M}$.

\section{BIBLIOGRAPHIE}

B ouch ard, R., Ga l my n, de M.-M. (1997), Médiation verbale et processus rédactionnel: parler pour écrire ensemble. In: Grossen, Michèle, Py, Bernard (éds). Pratiques sociales et médiations symboliques. Bern, Peter Lang. 153-173.

Clark, H. H. (1996), Using Language. Cambridge, Cambridge University Press.

Dausendschön-Gay, U., Krafft, U. (1991), Tâche conversationnelle et organisation du discours. In: Dausendschön-Gay, U., Gülich, E., Krafft, U. (éds). Linguistische Interaktionsanalysen. Beiträge zum 20. Romanistentag 1987. Tübingen, Niemeyer, 131-154.

Dausendschön-Gay, U., Krafft, U. (1991a), Rôles et faces conversationnels: à propos de la figuration en situation de contact. In: Russier, C., Stoffel, H., Véronique, D. (éds). Interactions en langue étrangère. Aix-en-Provence, PUP, 37-48.

Dausend schön-Gay, U., Güli ch, E., Krafft, U. (1992), Gemeinsam schreiben. Konversationelle Schreibinteraktionen zwischen deutschen $u$ nd französischen Gesprächspartnern. In: Krings, H.-P., Antos, G. (éds). Textproduktion. Neue Wege der Forschung. Trier, Wissenschaftlicher Verlag. 219-255.

${ }^{20} \mathrm{Ce}$ rôle n'existe pas lorsque chaque interactant inscrit sa propre version du texte. 
Dausendschön-Gay, U., Krafft, U. (1996), Prozesse interaktiven Formulierens: Konversationelles Schreiben in der Fremdsprache. In: Bömer, W., Vogel, K. (éds). Texte im Fremdsprachenerwerb. Verstehen und Produzieren. Tübingen, Narr. 253-274.

Gülich, E. (1991), Pour une ethnométhodologie linguistique: description de séquences conversationnelles explicatives. In: Dausendschön-Gay, U., Gülich, E., Krafft, U. (éds). Linguistische Interaktionsanalysen. Beiträge zum 20. Romanistentag 1987. Tübingen, Niemeyer, 325-364.

Gülich, E., Kotschi, Th. (1995), Discourse production in Oral Communication. In: Quasthoff, Uta M. (éd.). Aspects of Oral Communication. Berlin-New York, Walter de Gruyter, 30-66.

Herit age, J. (1995), Conversation Analysis. Methodological Aspects. In: Quasthoff, Uta M. (éd.). Aspects of Oral Communication. Berlin/New York, Walter de Gruyter, 391-418.

Krafft, U., Dau send schön-Gay, U. (1993), La coordination des activités conversationnelles: types de contrat. In: Actes du XX $X^{e}$ Congrès International de Linguistique et Philologie Romanes, tome II, section II. Tübingen, Niemeyer, 97-108.

K r aff t, U., D a us end schön-G ay, U. (1997), Les rédactions conversationnelles: construire ensemble un modèle de texte. In: Grossen, M., Py, B. (éds). Pratiques sociales et médiations symboliques. Bern, Peter Lang, 175-202.

$\mathrm{Krafft}, \mathrm{U}$., Dausend schön-Gay (sous presse), Système écrivant et processus de mise en mots dans les rédactions conversationnelles. Langages. 